\title{
Transfer Function Analysis of the Micro Cantilever Used in Atomic Force Microscopy
}

\author{
F. Javier Rubio-Sierra, Rafael Vázquez, and Robert W. Stark
}

\begin{abstract}
Current approaches to study the system response of the different modes of dynamic atomic force microscopy (AFM) use model simplification such as single mode approximations or are based on the nontrivial solution of the equation of motion. As an alternative to these approaches, the transfer function analysis gives a more complete description of system dynamics considering the cantilever as an extended vibrating structure. In order to describe the dynamics correctly, the precise experimental configuration has to be taken into account. This includes distinguishing between point loads (mechanical tip sample interaction) and distributed loads induced, e.g., by inertial driving or electrostatic forces. In this work we focus on a transfer function study of two different AFM configurations, the point force and distributed force driven cantilever. Exact analytical expressions of the infinite dimensional transfer function are derived for both possible system outputs: cantilever deflection and slope, which correspond to an interferometric and a light lever deflection detection system, respectively. Frequency response and transfer function infinite product expansion are obtained for the case where system outputs are set at the free end of the cantilever. In the frequency response, it is reflected the full complexity of cantilever dynamics affected by the presence of an infinite number of poles and zeros. An analytical expression for all the zeros and poles of the system is obtained. Using the transfer function, system dynamics are analyzed obtaining modal and antiresonant cantilever shapes along with its step response. From the frequency response, pole-zero investigations, and system dynamics, it is shown that both cantilever actuation and output measurement affect AFM operation. Transfer function analysis of AFM cantilevers improves the possibility of model-based AFM operation to increase imaging and manipulation performance.
\end{abstract}

Index Terms-Atomic force microscopy, microelectromechanical devices, nanotechnology, transfer functions.

\section{INTRODUCTION}

$\mathbf{T}$ HE ATOMIC force microscope (AFM) has played a vital role in the development of nanotechnology after its invention in 1986 by Binnig et al. [1]. As a microscope, the AFM is not only capable to image surfaces with atomic resolution as it can also be used to obtain quantitative local material properties such as roughness, visco-elasticity, composition of different materials ranging from semiconductor to biological samples [2], or force spectroscopy of single molecules [3]. Although the AFM

Manuscript received March 10, 2006; revised May 16, 2006. This work was supported by the European Commission under Contract NMP4-CT-2004-013684. The review of this paper was arranged by Associate Editor R. Lake.

F. J. Rubio-Sierra and R. W. Stark are with the CeNS and Section Crystallography in the Department of Earth and Environmental Sciences, University of Munich, 80333 Munich, Germany (e-mail: rubio@ lrz.uni-muenchen.de, stark@1mu.de).

R. Vázquez is with the MAE Department at the University of California at San Diego. La Jolla, CA 92037 USA (e-mail: rvazquez@ucsd.edu).

Digital Object Identifier 10.1109/TNANO.2006.883479 was originally conceived as a high-resolution imaging tool, it was rapidly extended to a tool for manipulation at the nanoscale [4]. Due to its versatility, the AFM offers a plethora of possibilities for surface modifications such as local anodic oxidation, chemical surface modification, or mechanical nanomachining, only to mention a few [5].

The dynamic operation of the AFM has emerged as a standard method both for imaging and manipulation. There are several different approaches to model the dynamics of an AFM cantilever. The most straightforward method relies on modeling the cantilever as a single-degree-of-freedom harmonic oscillator, the so-called first mode approximation [6]. However, the first mode approximation neglects that the cantilever is a distributed parameter system with an infinite number of resonant frequencies. The equation of motion of the AFM cantilever can be solved by analytical methods [7]. The cantilever also may be modeled as a lumped parameter system [8] parting from the analytical solution. However, if damping or tip-sample interaction are to be considered, the solution of the problem is not trivial [9].

Here, an analytical transfer function analysis of a generic cantilever beam may provide additional insight. This analysis method has successfully been applied to study the dynamics of flexible structures [10]. From the transfer function it is straightforward to obtain the frequency response, modes of vibrations, and step response of the system. Transfer functions of distributed parameter systems are transcendental functions. In order to obtain system poles and zeros, infinite product expansions must be used [11]. The analysis of poles and zeros facilitates the study of consequences in modeling and control design of the different inputs and outputs of the system [12].

In this work, the dynamics of a generic rectangular AFM cantilever are analyzed by the transfer function method. Analytical expressions of the transfer function for different system configurations are derived and the corresponding frequency responses are studied. Poles and zeros, which have both important implications in system stability and behavior, have been obtained from the infinite product expansion of the transfer functions. Using the analytical expression of the transfer function, the resonant and antiresonant responses of the different configurations are examined. Point and distributed force actuation of an AFM cantilever are studied along with the implications of tip deflection or slope measurement. This distinction between deflection or slope is important, since their sensitivity differs numerically in each of the vibration modes [8]. Finally, the step response of the AFM cantilever for the different system configurations is discussed. 


\section{MODELING}

\section{A. System Inputs and Outputs}

The microcantilever can be described by a transfer function representation by representing the cantilever as an isolated input/output system that is linear and time invariant. The inputs and outputs of the system to be considered in this work are the following.

Input 1 accounts for forces acting at the free end of the cantilever; these forces may be the interaction forces between tip and sample or a magnetic excitation realized by attaching a magnetic particle at the free end of the cantilever which is subject to a strong time varying magnetic field gradient [13]. Input 2 is a distributed uniform force along the cantilever; this corresponds to the inertial force caused by the usual mechanical excitation by a piezoelectric transducer below the cantilever chip [14] or to an electrostatic force.

Output 1 is the vertical deflection of the free end of the cantilever, usually measured by interferometric methods [15]. Output 2 is the deflection slope at the free end of the cantilever. This is the output measured in most of AFM systems using the light lever detection method [16].

In the next sections, we will consider the following system configurations: 1) case 11 , input 1 and output $1 ; 2$ ) case 12 , input 1 and output 2;3) case 21, input 2 and output 1 ; and 4) case 22, input 2 and output 2 .

\section{B. Dynamic Model of the System}

For the case of a generic rectangular beam the dynamics can be derived by using the classical Euler-Bernoulli beam model neglecting rotary inertia, shear deformation, axial effects, and the tip mass

$$
E I z_{x x x x}(x, t)+c \dot{z}(x, t)+m \ddot{z}(x, t)=-u(t)
$$

where the subindex $x$ denotes the partial derivative with respect to distance $(\partial / \partial x)$, and the dot differentiation with respect to time $(\partial / \partial t)$. The function $z(x, t)$ is the transversal displacement of the cantilever relative to its support, $u(t)$ is an uniform force per unit length acting along the cantilever, such as an inertial force caused by cantilever base displacement [7], $t$ denotes the time, $x \in(0, L)$ the longitudinal position along the cantilever, $L$ the length of the cantilever, $E I$ the flexural stiffness, $c$ the damping factor, and $m$ the mass per unit length. Note that the damping factor corresponds to, firstly, the intrinsic damping and secondly, the damping caused by the surrounding media. If the cantilever base is not moved, then both damping factors are combined in $c$. For the special case where the cantilever base is moved to induce cantilever vibrations, then a first order approach must be assumed [17] and the damping factor in (1) is given by $c\left(\dot{z}(x, t)+\dot{z}_{b}(t)\right)$, where $z_{b}(t)$ corresponds to the position of the cantilever base.

The boundary conditions at the fixed end of the tip are zero deflection and zero slope, namely,

$$
z(0, t)=0, \quad z_{x}(0, t)=0
$$

At the free end we consider no torque and a force $q(t)$ which is acting perpendicularly to cantilever axis. Then, both remaining boundary conditions are

$$
z_{x x}(L, t)=0, \quad E I z_{x x x}(L, t)=-q(t) .
$$

In order to obtain a transfer function description of the system we take the Laplace transform on (1), disregarding initial conditions

$$
E I Z_{x x x x}(x, s)+\left(c s+m s^{2}\right) Z(x, s)=-U(s)
$$

where $Z(s, x)$ and $U(s)$ represent the Laplace transform of the cantilever transversal position and the distributed force along the cantilever, and $s$ is the (complex) Laplace variable. The boundary conditions in the Laplace domain are given by

$$
\begin{aligned}
Z(0, s) & =0, \quad Z_{x}(0, s)=0, \\
Z_{x x}(L, s) & =0, \quad E I Z_{x x x}(L, s)=-Q(s)
\end{aligned}
$$

where $Q(s)$ is the Laplace transform of the force at the free end of the cantilever.

Equation (4) can be solved using the theory of linear ordinary differential equations [18]. We define

$$
\lambda(s)=\sqrt[4]{\frac{c s+m s^{2}}{4 E I}} .
$$

In the following, we will omit the dependence of $\lambda$ on $s$ for simplicity of notation. The solution for the homogeneous equation is

$$
\begin{aligned}
Z_{h}(x, s)=e^{\lambda x}\left[C_{1} \sin (\lambda x)+C_{2} \cos (\lambda x)\right] & \\
& +e^{-\lambda x}\left[C_{3} \sin (\lambda x)+C_{4} \cos (\lambda x)\right]
\end{aligned}
$$

where the constants $C_{1}, C_{2}, C_{3}$, and $C_{4}$ can be found substituting (7) into the boundary conditions (5). For the nonhomogeneous case, the general solution is of the form

$$
Z(x, s)=Z_{h}(x, s)+W(x, s)
$$

where $W(x, s)$ is a particular solution of the nonhomogeneous equation. For the first force input, we define the transfer function from the point force to cantilever deflection $G_{11}$ and from the point force to cantilever slope $G_{12}$ as

$$
G_{11}(L, s)=\frac{Z(L, s)}{Q(s)}, \quad G_{12}(L, s)=\frac{Z_{x}(L, s)}{Q(s)}
$$

where $U(s)=0$ is considered. Likewise, we define for the second input the transfer function from the distributed force to cantilever deflection, $G_{21}$, and from distributed force to cantilever slope, $G_{22}$, as

$$
G_{21}(L, s)=\frac{Z(L, s)}{U(s)}, \quad G_{22}(L, s)=\frac{Z_{x}(L, s)}{U(s)}
$$

considering $Q(s)=0$. For the special case where the cantilever base is moving, the enviromental damping has to be considered in the Bernoulli equation as mentioned above. For this case, the moving base introduces a multiplying factor in the transfer 
functions calculated from (1). The transfer function considering a moving base, and using the transfer function of the unmoved base $G_{i j}(s)$, is then given by

$$
G_{i j}^{\prime}(s)=G_{i j}(s) \frac{(c+m s)}{(m s)} .
$$

Once the transfer function has been calculated, it is straightforward to obtain the frequency response of the system. At a given angular frequency $\omega$, the response amplitude is given by $|G(j \omega)|$, while the phase response is given by $\angle G(j \omega)$; that is, the modulus and angle of the complex-valued function $G(s)$ evaluated on the imaginary axis. The frequency response of the cantilever at a given point can then be displayed using the Bode plot representation [19].

All numerical data and plots obtained from the transfer functions in this chapter have been obtained using the parameters of a commercial cantilever [20]: $E=169 \mathrm{GPa}, m=4.08 \times$ $10^{-7} \mathrm{~kg} / \mathrm{m}, L=232 \mu \mathrm{m}, w=40 \mu \mathrm{m}, d=4 \mu \mathrm{m}$, and $c=0.01 \mathrm{~kg} / \mathrm{ms}$, where $w$ and $d$ are cantilever width and thickness, respectively.

\section{Point Force at the Free End}

At first, we consider the case where the input is a point load $q(t)$ acting at the free end of the cantilever without distributed forces $(u(t)=0)$. To obtain the solution of the equation of motion, it is only necessary to substitute the boundary conditions (5) in the homogeneous solution (7), thus obtaining a linear system of equations in the constants $C_{1}, C_{2}, C_{3}$, and $C_{4}$. The solution is then

$$
\begin{aligned}
Z^{\text {point }}(x, s)= & \frac{-2 Q(s)}{E I \lambda^{3} D(s)} \\
& \times[\cos (\lambda L) \cosh (\lambda(L-x)) \sin (\lambda x) \\
& -\cos (\lambda(L-x)) \cosh (\lambda x) \sinh (\lambda x)]
\end{aligned}
$$

where

$$
D(s)=2[2+\cos (2 \lambda L)+\cosh (2 \lambda L)] .
$$

We can directly obtain the transfer functions for the different outputs considered. The transfer function from the punctual force to tip position is obtained by substituting $x=L$ in the previous equation, which gives

$$
G_{11}(L, s)=\frac{\sinh (2 \lambda L)-\sin (2 \lambda L)}{E I \lambda^{3} D(s)} .
$$

To obtain the transfer function $G_{12}(L, s)$ from the punctual force to cantilever slope at the free end, it is necessary, firstly, to take the spatial derivative of the solution, and then to make the same substitution as before. The obtained transfer function is

$$
G_{12}(L, s)=\frac{\cosh (2 \lambda L)-\cos (2 \lambda L)}{E I \lambda^{2} D(s)} .
$$

The Bode plots for both transfer functions are shown in Fig. 1 The dynamic response for both outputs presents alternating resonances and antiresonances. The resonances are located at the
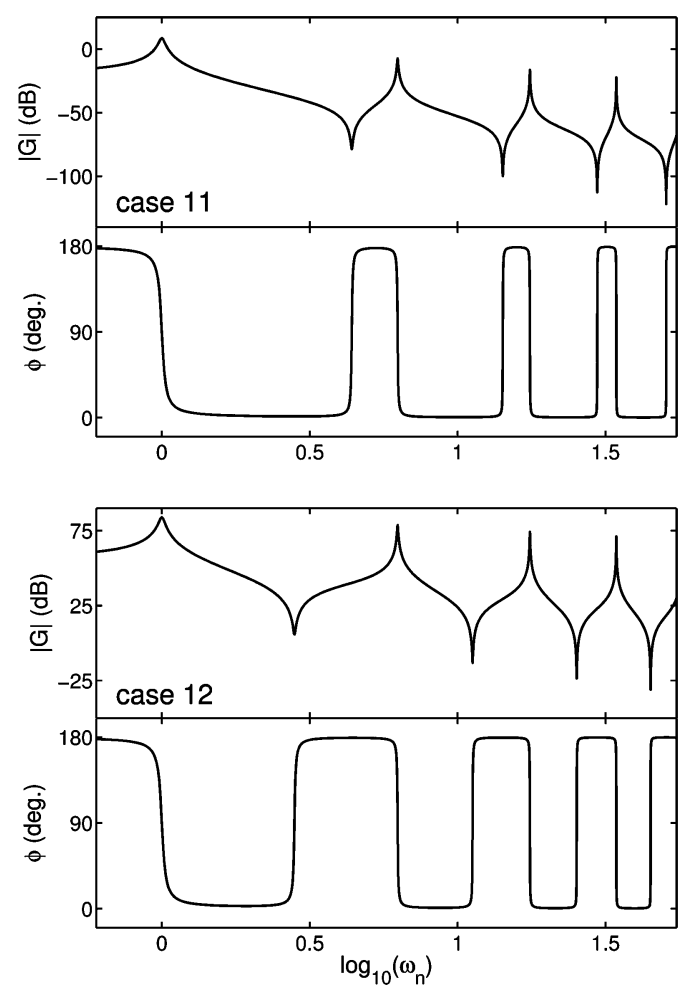

Fig. 1. Bode plot of the response of a cantilever to a point force applied at its end. The frequency $\omega_{n}$ has been normalized with respect to the first resonant frequency of the system.

same frequencies for both outputs due to the common denominator. The antiresonances frequencies are different for both cases. At the resonances the phase decreases by $180^{\circ}$ whereas at the antiresonances the phase is increased by $180^{\circ}$.

\section{Distributed Force}

Next we consider the case where the cantilever is driven by a distributed force and $q(t)=0$. In this case, (4) is nonhomogeneous. A particular solution is given by

$$
W(x, s)=\frac{U(s)}{c s+m s^{2}} .
$$

Again, we substitute the general solution into the boundary conditions (5) to obtain the coefficients from (8). The solution is

$$
\begin{aligned}
Z^{\mathrm{dist}}(x, s)=\frac{-}{2 E I} & I(s) \\
\times[ & D(s)-\cosh (\lambda x) \cos (\lambda(2 L-x)) \\
& -(\cosh (\lambda(2 L-x)) \\
& +2 \cosh (\lambda x)) \cos (\lambda x) \\
& +\sinh (\lambda x) \sin (\lambda(2 L-x)) \\
& +\sinh (\lambda(2 L-x)) \sin (\lambda x)] .
\end{aligned}
$$

The transfer function from the distributed force per unit length to tip position is

$$
G_{21}(L, s)=\frac{-1}{E I \lambda^{4}} \frac{[\cosh (\lambda L)-\cos (\lambda L)]^{2}}{D(s)} .
$$



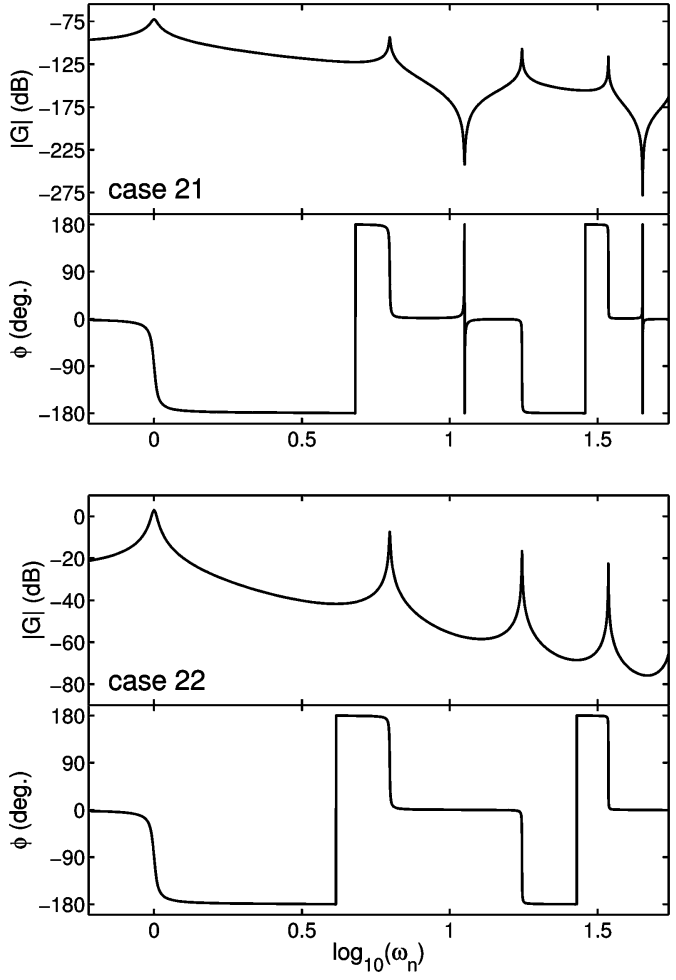

Fig. 2. Bode plot of the response of a cantilever to a distributed force. Sharp edges at the position response represent phase increments of $360^{\circ}$; in the slope response, the sharp edges are due to the continuity of the phase between $-180^{\circ}$ and $180^{\circ}$. The frequency $\omega_{n}$ has been normalized with respect to the first resonant frequency of the system.

Again, by spatial differentiation of the solution we obtain the transfer function for the second output

$$
G_{22}(L, s)=-2 \frac{\cosh (\lambda L) \sin (\lambda L)-\cos (\lambda L) \sinh (\lambda L)}{E I \lambda^{3} D(s)} .
$$

The frequency response for both transfer functions is shown in Fig. 2. The denominators of both transfer functions are the same as for the point load. Therefore, the resonances are located at the same frequencies. The antiresonances of the position frequency response alternate each two resonance peaks, while there are not any antiresonances in the slope response. At the antiresonances of the position response, there is a phase increment of $360^{\circ}$ (sharp edges at the phase diagram). In the slope response, there is a decrease of $90^{\circ}$ at the resonances.

Note from (14), (15), (18), and (19) that the poles of the cantilever transfer function are the same for both inputs and outputs considered and do not depend on the actuator and sensor position as is usual in dynamical control systems: the location of sensors and actuators can change the position of zeros, but not the denominator (the poles), which are "inherent" to the dynamics of the system.

\section{E. Poles of the Transfer Functions}

The common denominator $D(s)$ can be factorized by direct application of the infinite product expansions table given in
Goodson [11] and by using some trigonometric relations. The result is

$$
\begin{aligned}
D(s) & =8 \prod_{n=1}^{\infty}\left[1+\frac{4 \lambda^{4} L^{4}}{c_{n}^{4}}\right], \\
\cos c_{n} \cosh c_{n} & =-1, \quad c_{n}>0, \text { real. }
\end{aligned}
$$

The s-plane roots of $D(s)$ are the poles of the system, whose characterization is important for control analysis and to obtain an expression for all the resonant frequencies of the system. By substitution of (6) in (20), the fourth power of lambda in (20) reduces the fourth root in (6) and we find, for each $n$, a (simple) complex conjugate pair of imaginary poles in the s-plane

$$
s_{n}^{\text {pole }}=-\frac{c}{2 m} \pm \sqrt{\frac{c^{2}}{4 m^{2}}-\frac{E I c_{n}^{4}}{m L^{4}}} .
$$

All the poles are located in the left half plane (LHP), which means that the damped cantilever is stable. The numerical values for the first three conjugate pairs are

$$
\begin{aligned}
& s_{1}^{\text {pole }}=-13.4 \times 10^{3} \pm 0.64 \times 10^{6} i\left(\frac{\mathrm{rad}}{\mathrm{s}}\right) \\
& s_{2}^{\text {pole }}=-13.4 \times 10^{3} \pm 4.02 \times 10^{6} i\left(\frac{\mathrm{rad}}{\mathrm{s}}\right) \\
& s_{3}^{\text {pole }}=-13.4 \times 10^{3} \pm 1.12 \times 10^{7} i\left(\frac{\mathrm{rad}}{\mathrm{s}}\right)
\end{aligned}
$$

To each complex conjugate pair of poles corresponds a resonant frequency. Note that if base motion is being considered, then (11) introduces an additional pole at $s=0$.

An analytical expression for each resonant frequency is not easy to obtain from the value of the poles, but since the damping of each pole (the negative real part) is very small (compared to the modulus of the pole), it can be considered to be approximately at the imaginary part of the conjugate pair of poles

$$
\omega^{\mathrm{res}}(n)=\operatorname{Im}\left(s_{n}\right)
$$

For our case study, the first three resonances of the system are: $102 \mathrm{kHz}, 640 \mathrm{kHz}$, and $1.79 \mathrm{MHz}$.

\section{F. Amplitude and Phase Lag Along the Cantilever}

Once the resonant frequencies are known, it is straightforward to obtain the amplitude and phase response of the transfer function along the cantilever in the natural modes of vibration using (12) and (17). The amplitude and slope response in the first three natural modes for all case studies are shown in Fig. 3. The amplitude response for both inputs is equivalent while they differ in the phase response. Resonant modes of vibration are of great importance for AFM measurements, since they enable frequency and amplitude modulated operation of the cantilever [21]. From the resonant response of the cantilever, it is observed that the gradient of the response at the end part of the cantilever, where system output is measured in standard AFM systems, is smaller for the second output, which enhances system output stability against internal drift of the microscope components. 

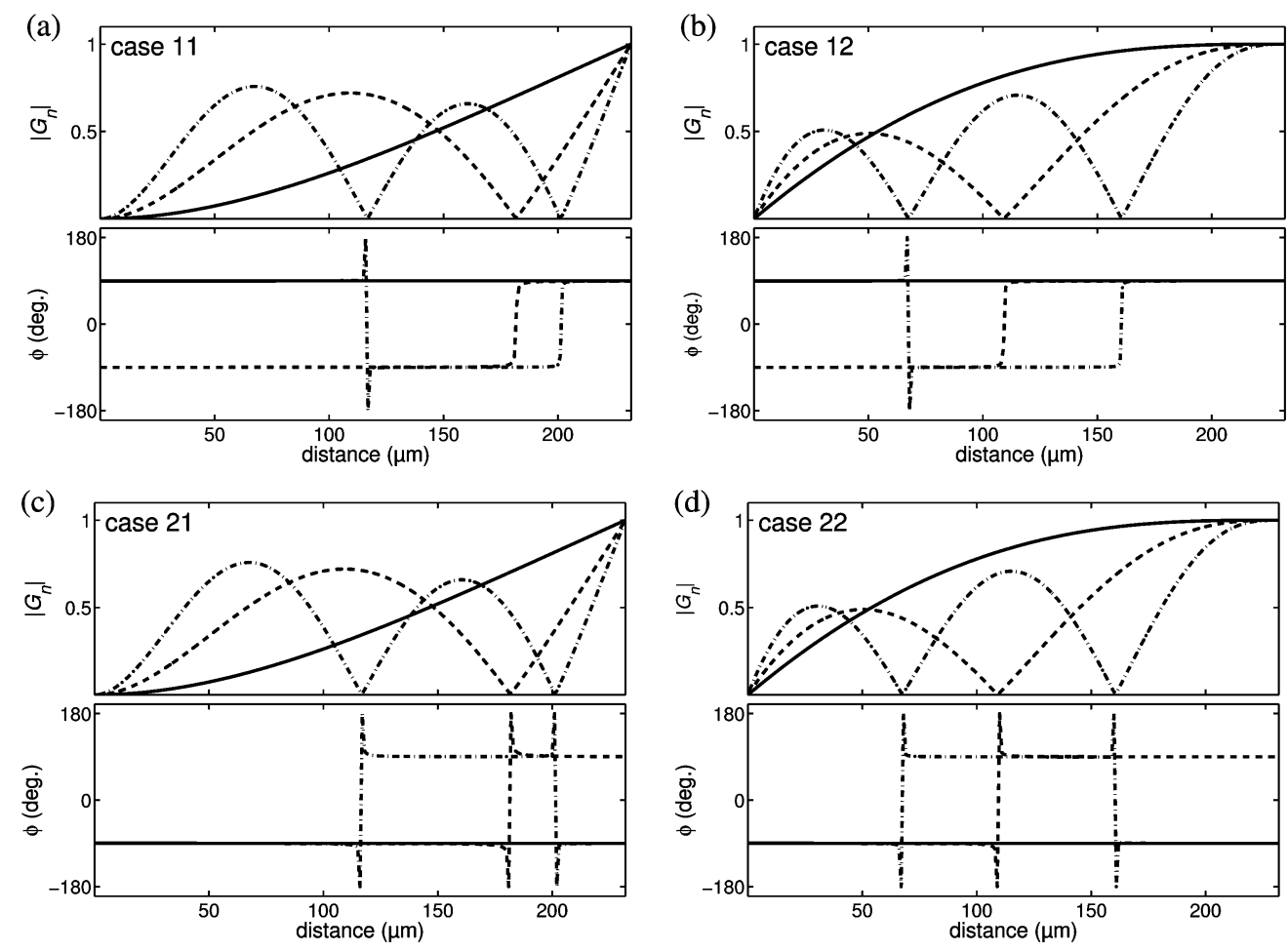

Fig. 3. Magnitude and phase response along the cantilever at the first three resonant frequencies: (a) case 11; (b) case 12; (c) case 21; and (d) case 22. The magnitude $\left|G_{n}\right|$ has been normalized to the maximum of each mode.

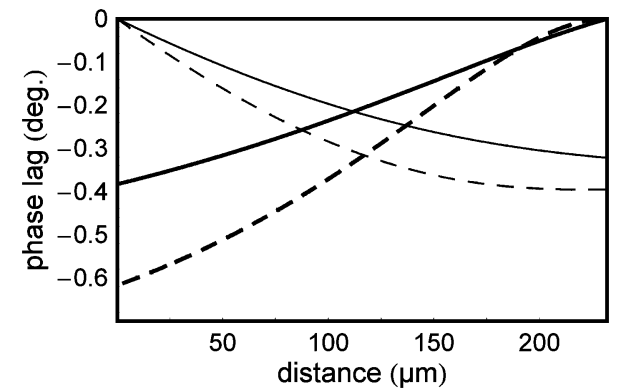

Fig. 4. Phase lag along the cantilever for the four different cases: thick solid line, case 11; thick dashed line, case 12; thin solid line, case 21 ; thin dashed line, case 22 .

Due to the finite velocity of propagation of the bending waves along the cantilever, there is a phase variation between the resonance nodes. The phase lag is given by

$$
\Delta \phi=-\frac{\omega \Delta x}{\nu}
$$

where $\Delta \phi$ is the change in phase of the bending wave traveling a distance $\Delta x$ with propagation velocity $\nu$. The velocity of propagation is not constant because the dispersive nature of the flexural vibrations of the cantilever [7]. Fig. 4 shows the phase lag along the cantilever for the different inputs and outputs. As expected, for the point load case, the phase lag increases from the free end of the cantilever to its base, in the opposite direction as in the distributed force case.

\section{G. Zeros of the Transfer Function}

Factorization of the numerators of (14), (15), (18), and (19) is practically impossible except in the case where $x=L$. In this case, transcendental functions whose product expansions are found in the literature [11] can be easily obtained either directly or after the use of some trigonometric relations. The infinite product expansions of the four different transfer functions are

$$
\begin{aligned}
& G_{11}(L, s)=\frac{8 L^{3}}{3 E I D(s)} \prod_{n=1}^{\infty}\left[1+\frac{4 \lambda^{4} L^{4}}{d_{n}^{4}}\right], \\
& G_{12}(L, s)=\frac{4 L^{2}}{E I D(s)} \prod_{n=1}^{\infty}\left[1+\frac{4 \lambda^{4} L^{4}}{n^{4} \pi^{4}}\right], \\
& G_{21}(L, s)=\frac{-L^{4}}{E I D(s)}\left(\prod_{n=1}^{\infty}\left[1+\frac{\lambda^{4} L^{4}}{4 n^{4} \pi^{4}}\right]\right)^{2}, \\
& G_{22}(L, s)=\frac{-4 L^{3}}{3 E I D(s)} \prod_{n=1}^{\infty}\left[1-\frac{\lambda^{4} L^{4}}{d_{n}^{4}}\right],
\end{aligned}
$$

where

$$
\tan d_{n}=\tanh d_{n}, \quad d_{n}>0, \text { real. }
$$

From these expressions it is straightforward to obtain an exact numerical expression for the zeros of the transfer functions proceeding similarly as when we obtained the poles of the transfer function. Fig. 5 shows a graphical representation for the first three pairs of zeros of each output of both case studies. In this figure it is clearly seen that the transfer functions corresponding to cases 11,12 , and 21 have all their complex conjugate pairs of zeros in the LHP. For case 22, the complex conjugate pairs of zeros are all in the real axis and alternate in the LHP and right half plane (RHP).

The point load configuration corresponds to a system where the measurement is collocated (sensor and actuator in the same position). Therefore, it was expected to find all the zeros of 


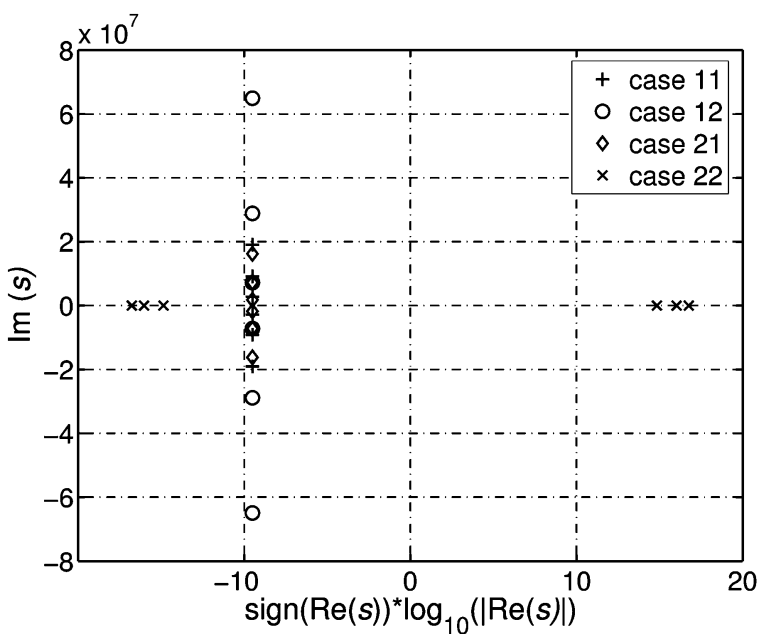

Fig. 5. First three complex conjugate zeros of the four different transfer functions from (25)-(28).

TABLE I

FIRST THREE ANTIRESONANT FREQUENCIES

\begin{tabular}{l||c||c}
\hline Case 11 & Case 12 & Case 21 \\
\hline \hline $448 \mathrm{kHz}$ & $287 \mathrm{kHz}$ & $197 \mathrm{kHz}$ \\
\hline $1.45 \mathrm{MHz}$ & $1.15 \mathrm{MHz}$ & $4.72 \mathrm{MHz}$ \\
\hline $3.03 \mathrm{MHz}$ & $2.58 \mathrm{MHz}$ & $10.6 \mathrm{MHz}$ \\
\hline
\end{tabular}

the system in the LHP. This has some important repercussions for the dynamics and control of the cantilever: if no zeros are present in the RHP, it is easier to stabilize the system and modal truncation accurately simulates the dynamics of the distributed system [8].

The configuration for the second input is noncollocated (sensors and actuators in different position). The position measurement (case 21) does not have RHP poles, and therefore is a minimum phase system. However, as observed in the Bode plot of Fig. 2, the phase contribution exceeds $-180^{\circ}$ after each two resonant frequencies, and recovering $360^{\circ}$ in the antiresonant frequency. This must be taken into account for control design. The system where the slope is used as output (case 22) suffers from presence of RHP zeros and after the first resonance the phase contribution always exceeds $-180^{\circ}$, and hence its transfer function is nonminimum phase. Therefore, in this configuration the system is subject to performance limitations and is more difficult to control than in the other considered configurations [12].

For the special case where cantilever base motion is present in the system, then (11) introduces an additional zero at $s=$ $-c / m$, i.e., an LHP zero which does not modify the quantitative analysis of the system presented above.

\section{H. Antiresonant Cantilever Response}

Once the infinite product expansions for the different transfer functions have been calculated, it is possible to obtain an approximation to the antiresonant frequencies from the imaginary part of each complex conjugate pair of zeros (with nonzero imaginary part). Different from the resonant frequencies, each system configuration has different values of the antiresonant frequencies. Table I shows the values of the first three antiresonant frequencies for each case. Case 22 does not have antiresonances. The first three antiresonance modes of vibration for every system configuration are shown in Fig. 6.

\section{Step Response of the Free Cantilever}

By application of the Laplace transform, the response of the system to a given input can be obtained as the product of the transfer function times the transformed input. Then, the time domain response can be recovered using the inverse Laplace transform. In particular, the step response of the system in the time domain $y_{\text {step }}(t)$ is obtained by direct application of inverse Laplace transform to the product of the system transfer function and the unit step 1/s [19]. The problem reduces to solve the following integral:

$$
y_{\text {step }}(t)=\frac{1}{2 \pi i} \int_{c-i \infty}^{c+i \infty} e^{s t} \frac{G(s)}{s} .
$$

This integral can be evaluated by direct application of the residue theorem [22]

$$
y_{\text {step }}(t)=\sum_{k=0}^{\infty} \operatorname{Res}\left[e^{s t} \frac{G(s)}{s}\right]_{s=s_{k}}
$$

where $s_{k}$ are the singular points, i.e., the poles, of the function where the residues are evaluated. Since the poles of the transfer function are known, the problem is reduced to calculate and sum the residues. In the sum only the first $n$ poles contributing to the system response will be considered, since we found the contribution of high order poles to be rapidly diminishing, i.e., the series (31) converges very fast, so its truncation is an accurate estimate of the sum. The four transfer functions considered above have no poles at zero. Thus, the integrand in (30), where the residues are evaluated, has a single pole at $s=0$ corresponding to the step response transfer function. The rest of the poles are given by the infinite product expansion of the transfer function denominator (21). Taking into account these considerations, we can write

$$
y_{\text {step }}(t)=G(0)+\sum_{k=1}^{\infty} \frac{e^{s_{k} t}}{s_{k}} \operatorname{Res}[G(s)]_{s=s_{k}} .
$$

$G(0)$ is easily evaluated using the expressions for numerators and common denominator presented above [(20), (25)-(28)]. The residues of the transfer function can be evaluated as

$$
\operatorname{Res}[G(s)]_{s=s_{k}}=N\left(s_{k}\right) \lim _{s \rightarrow s_{k}} \frac{s-s_{k}}{D(s)}=\frac{N\left(s_{k}\right)}{D^{\prime}\left(s_{k}\right)}
$$

where $N\left(s_{k}\right)$ and $D\left(s_{k}\right)$ are the numerator and denominator of the transfer function evaluated at $s_{k}$ respectively. In (33), the L'Hôpital rule [23] has been applied to solve the limit and $D^{\prime}\left(s_{k}\right)$ denotes the derivative of the denominator with respect to $s$. Introducing (33) in (32) and applying the chain rule, we find the general expression of the unit step response as

$$
y_{\text {step }}(t)=G(0)+\sum_{n}^{k=1} \frac{e^{s_{k} t}}{s_{k}} \frac{N\left(\lambda\left(s_{k}\right)\right)}{D^{\prime}\left(\lambda\left(s_{k}\right)\right) \lambda^{\prime}\left(s_{k}\right)} .
$$

If the cantilever base motion is being considered then (11) must be used to calculate the step response. 

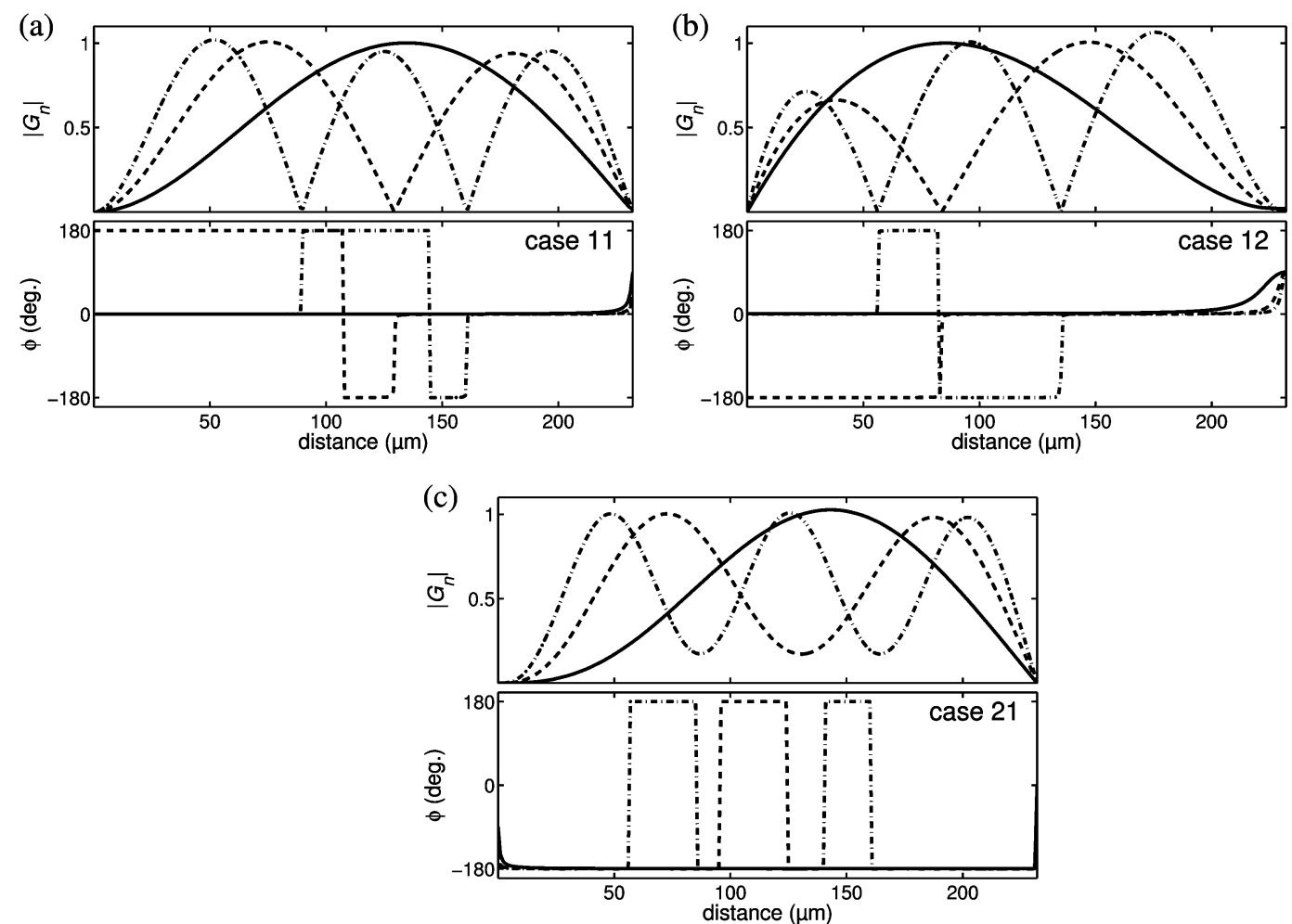

Fig. 6. Magnitude and phase response along the cantilever at the first three antiresonant frequencies: (a) case 11; (b) case 12; and (c) case 21. The magnitude $\left|G_{n}\right|$ has been normalized to the maximum of each mode.
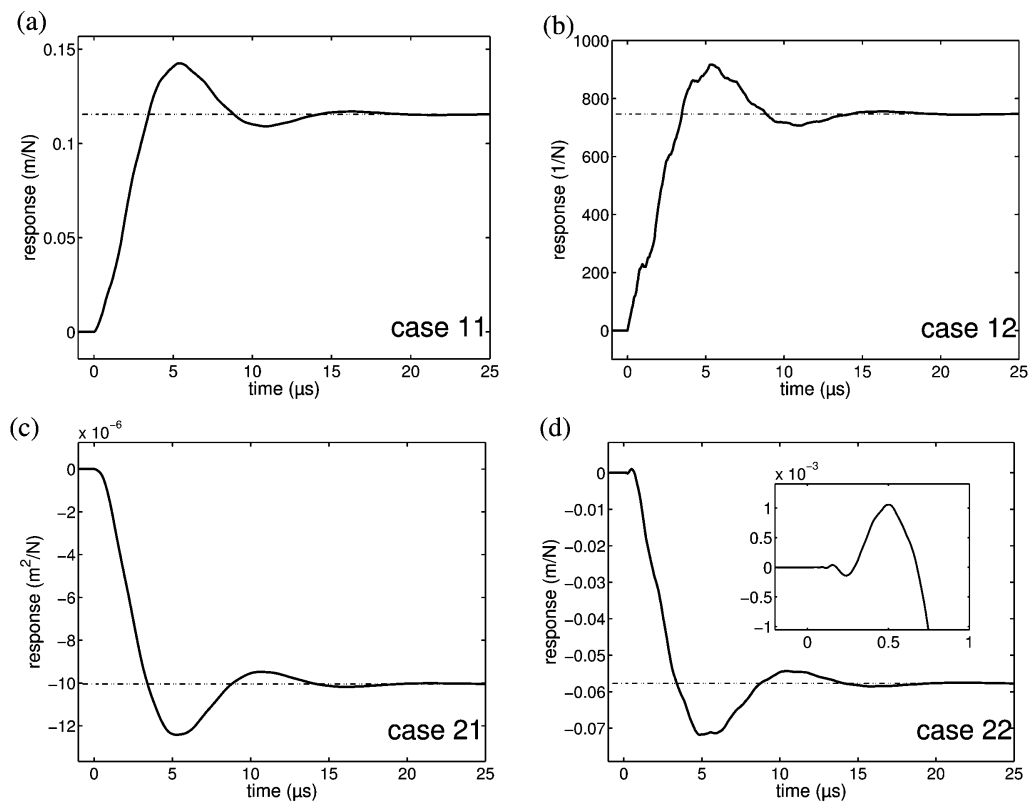

Fig. 7. Response of a strongly damped cantilever $(c=0.3 \mathrm{~kg} / \mathrm{ms})$ to a unit force step: (a) case 11; (b) case 12; (c) case 21; and (d) case 22. The insert in (d) shows a zoom of the initial response of the system in case 22 against the actuation due to its nonminimum phase behavior. Dotted lines correspond to the steady state solution of (1). The number of poles $n$ considered was of 20 .

Fig. 7 shows the response to an unit force step of the different system configurations considered above. The damping factor used was of $c=0.3 \mathrm{~kg} / \mathrm{ms}$, a value representative of an AFM cantilever working in liquid to better illustrate the dynamics of the system. The dotted line in each graph corresponds to the steady state solutions of (1). For the different system inputs, the step responses are in opposite directions. It is worth noting that in case 22, distributed force input and slope as output, the system reacts at the very beginning against the input. This nonintuitive behaviour of the cantilever is due to the fact that case 22 is a nonminimum phase configuration of the system. In this case, it is seen that the properties of the system transfer function gives direct insight in cantilever transient behavior. The step response calculated using simplified AFM dynamical models 
would not be accurate enough, since, as seen in (31), all modes contribute in the response. Models considering only a larger but finite number of modes will not get a much better result either, unless they accurately capture the zero dynamics of the system, which plays an important role in the response as seen in (34). Moreover, the study of the response of the AFM cantilever to a step force input has a direct application to the analysis of force spectroscopy curves obtained by the AFM [24].

\section{CONCLUSION}

In this work we have presented an analytic approach to study the dynamics of a free vibrating AFM cantilever. The transfer functions for two different system outputs, cantilever deflection and slope along the cantilever, have been obtained. Two different case studies, corresponding both different inputs, point and distributed force actuation, have been analyzed. From the transfer functions, frequency response and infinite product expansion for the particular case of outputs measured at the end of the cantilever have been obtained. In the first case, frequency response analysis shows the presence of resonances and antiresonances and the phase never decreases more than $180^{\circ}$. In the second case, the position output has also antiresonances and its phase shift does not exceed $360^{\circ}$, while for the slope output no antiresonances are present and is nonminimum in phase. From the infinite product expansions, exact expressions for the poles and zeros in all the cases have been obtained. Only the transfer function in the second case with cantilever slope as output suffers the presence of RHP zeros. To illustrate direct applications of the transfer function with important implications on AFM operation, the resonant and antiresonant response along with the step response of the cantilever have been obtained.

The transfer function method can be extended to describe other input forces such as electrostatic actuation, where the distributed force is not constant along the cantilever, or tip-sample interaction in the linear approximation. Transfer function analysis also opens the possibility of model-based control and observer design for AFM cantilevers to improve state-of-the-art performance in microscopy, force spectroscopy, and nanomanipulation.

\section{ACKNOWLEDGMENT}

The authors would like to thank Prof. M. Krstic and Prof. W. M. Heckl for their support of this work.

\section{REFERENCES}

[1] G. Binnig, C. F. Quate, and C. Gerber, "Atomic force microscopy," Phys. Rev. Lett., vol. 56, no. 9, pp. 930-933, 2003.

[2] R. J. Colton, A. Engel, J. E. Frommer, H. E. Gaub, A. Gewirth, R. Guckenberger, J. Rabe, W. M. Heckl, and B. Parkinson, Procedures in Scanning Probe Microscopies. Chichester, U.K.: Wiley, 1998.

[3] E. L. Florin, V. T. Moy, and H. E. Gaub, "Adhesion forces between individual ligand-receptor pairs," Science, vol. 264, pp. 415-417, 1994.

[4] D. Wouters and U. S. Schubert, "Nanolithography and nanochemistry: Probe-related patterning techniques and chemical modification for nanometer-sized devices," Angew. Chem. Int. Ed., vol. 43, no. 19, pp. 2480-2495, 2004
[5] F. J. Rubio-Sierra, W. M. Heckl, and R. W. Stark, "Nanomanipulation by atomic force microscopy," Adv. Eng. Mater., vol. 7, no. 4, pp. 193-196, 2005.

[6] N. A. Burnham, G. Gremaud, A. J. Kulik, P. J. Gallo, and F. Oulevey, "Materials' properties measurements: Choosing the optimal scanning probe microscope configuration," J. Vac. Sci. Technol. B, vol. 14, pp. 1308-1312, 1996.

[7] L. Meirovitch, Analytical Methods in Vibrations. New York: Macmillan, 1967.

[8] R. W. Stark, G. Schitter, M. Stark, R. Guckenberger, and A. Stemmer, "State space model of freely vibrating and surface-coupled cantilever dynamics in atomic force microscopy," Phys. Rev. B, vol. 69, no. 8, pp. 085412-1-085412-9, 2004.

[9] D. W. Dareing, T. Thundat, J. Sangmin, and M. Nicholson, "Modal analysis of microcantilever sensors with enviromental damping," $J$. Appl. Phys., vol. 97, no. 8, pp. 084902-1-084902-6, 2005.

[10] J. L. Junkins and Y. Kim, Introduction to Dynamics and Control of Flexible Structures. New York: American Institute of Aeronautics and Astronautics, 1993.

[11] R. E. Goodson, "Distributed system simulation using infinite product expansions," Simulation, vol. 15, no. 6, pp. 255-263, 1970.

[12] V. A. Spector and H. Flashner, "Modeling and design implications of noncollocated control in flexible systems," ASME J. Dynam. Syst., Measure. Control, vol. 112, no. 2, pp. 186-193, 1990.

[13] E. L. Florin, M. Radmacher, B. Fleck, and H. E. Gaub, "Atomic force microscope with magnetic force modulation," Rev. Sci. Instrum., vol. 65, no. 3, pp. 639-643, 1994.

[14] C. A. J. Putman, K. O. V. der Werf, B. G. D. Grooth, N. F. V. Hulst, and J. Greva, "Tapping mode atomic force microscopy in liquid," Appl. Phys. Lett., vol. 64, no. 18, pp. 2454-2456, 1994.

[15] H. J. Mamin, R. Erlandsson, J. E. Stern, and B. Terris, "Force microscope using a fiber-optic displacement sensor," Rev. Sci. Instrum., vol. 59, no. 11 , pp. 2337-2340, 1988 .

[16] G. Meyer and N. M. Amer, "Optical-beam-deflection atomic force microscopy: The $\mathrm{NaCl}$ (001) surface," Appl. Phys. Lett., vol. 56, no. 21, pp. 2100-2101, 1990.

[17] B. Anczykowski, B. Gotsmann, H. Fuchs, J. P. Cleveland, and V. B. Elings, "How to measure energy dissipation in dynamic mode atomic force microscopy," Appl. Surf. Sci., vol. 140, pp. 376-382, 1999.

[18] G. Simmons, Differential Equations With Applications and Historical Notes. New York: McGraw-Hill, 1991.

[19] G. F. Franklin, J. D. Powell, and A. Emami-Naeini, Feedback Control of Dynamic Systems. Reading, MA: Addison-Wesley, 1986.

[20] NanoWorld AG. Erlangen, Germany.

[21] R. Garcia and R. Perez, "Dynamic atomic force microscopy methods," Surf. Sci. Rep., vol. 47, no. 6, pp. 197-301, 2002.

[22] I. N. Bronstein, K. A. Semendjajew, G. Musiol, H. Musiol, and H. Mühlig, Taschenbuch der Mathematik. Frankfurt am Main, Germany: Harri Deutsch, 1977.

[23] T. M. Apostol, Calculus. New York: Wiley, 1967, vol. 1.

[24] B. Cappella and G. Dietler, "Force-distance curves by atomic force microscopy," Surf. Sci. Rep., vol. 34, pp. 1-104, 1999.

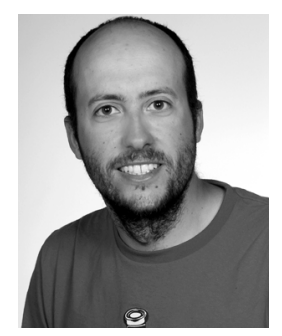

F. Javier Rubio-Sierra received a B.S. degree in physics from the University of Sevilla, Sevilla, Spain in 2000. He is currently working towards the Ph.D. degree in the Department of Earth and Environmental Sciences at the University of Munich, Munich, Germany.

$\mathrm{He}$ has worked for Sony Deutschland $\mathrm{GmbH}, \mathrm{Mu}-$ nich, Germany (2001-2002) measuring performance of telecommunication devices. He was a Marie Curie Fellow at the University of Munich (2002-2003), and participated in the International Graduate School "Nano-Bio-Technology" in the Center for Nanoscience in Munich, Germany (2004-2005). His main research fields are AFM design, nanomanipulation, and nanomechatronics. 


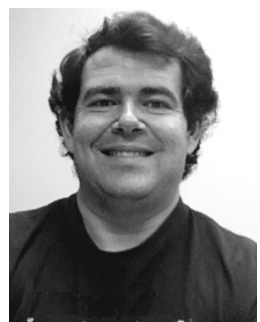

Rafael Vázquez received B.S. degrees in electrical engineering and mathematics from the University of Sevilla, Sevilla, Spain, in 1999 and 2003, respectively, and the M.S. degree in aerospace engineering from the University of California, San Diego, La Jolla, in 2004. He is currently working toward the $\mathrm{Ph} . \mathrm{D}$. degree in the Department of Mechanical and Aerospace Engineering, the University of California, San Diego, CA.

He has worked as systems engineer for Telvent Interactiva, Sevilla (2000-2001), and was a CTS Marie Curie Fellow at the Université Paris-Sud (2005). His research interests include dynamical systems, nonlinear control, control of distributed parameter systems, and applications to flow control and nanomechatronics.

Mr. Vazquez is also a student member of SIAM and ASME. He has been a finalist for the Best Student Paper Award in the 2005 CDC, Sevilla, Spain.

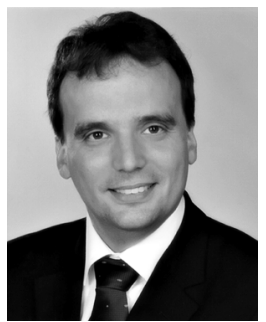

Robert W. Stark received the Diploma degree in physics and the Ph.D. degree in natural sciences from the University of Munich, Munich, Germany, in 1996 and 2001, respectively.

From 2001 to 2003, he was a Postdoc at the ETH Zurich in the Department of Mechanical and Process Engineering in the Nanotechnology Group. He was appointed a Junior Group Leader at the University of Munich in 2003, where he is now heading an independent research group working on topics related to tribology and nanomanipulation.

Dr. Stark is a full member of the Excellence Network NanoBiotechnology (ENNAB) in Munich and an extraordinary member of the Center for Nanoscience CeNS since 2003. He is also a member of the German Association of Engineers (VDI) and the German Physical Society (DPG). In 2002 he received the young researchers award "Nanotechnology" from the German Federal Ministry of Education and Research. 